\title{
Protective Signatures of Roselle (Hibiscus sabdariffa L.) Calyx Fractions against Staphylococcus aureus in Drosophila Infection Model
}

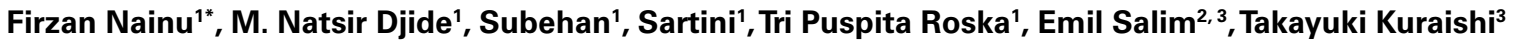 \\ ${ }^{1}$ Faculty of Pharmacy, Universitas Hasanuddin, Makassar, Indonesia \\ ${ }^{2}$ Faculty of Pharmacy, Universitas Sumatera Utara, Medan, Indonesia \\ ${ }^{3}$ Faculty of Pharmacy, Institute of Medical, Pharmaceutical and Health Sciences, Kanazawa University, Kanazawa, Ishikawa, Japan
}

\section{ARTICLE INFO}

Article history:

Received March 14, 2020

Received in revised form September 15, 2020

Accepted September 22, 2020

\section{KEYWORDS:}

Drug-resistant bacteria,

Antistaphylococcal,

Hibiscus sabdariffa,

roselle fractions,

Fruit fly model

\begin{abstract}
The rise of antibiotic-resistant Staphylococcus aureus-related clinical cases is an alarming chronicle for global communities. This research was conducted to examine the antistaphylococcal effect of roselle (Hibiscus sabdariffa L.) calyx fractions in the Drosophila model. In the infection experiment, wild-type and immunodeficient Drosophila were pricked with $S$. aureus and subsequently subjected to fly survivorship and colony-forming assays, in the presence or absence of roselle calyx fractions. The Involvement of immune stimulation in the host antibacterial protection was assessed in vitro using cell-based luciferase reporter assay and in vivo using RT-qPCR analysis on adult flies. A declining rate of fly survivorship and augmentation of bacterial growth were observable in $S$. aureusinfected wild-type flies but subject to improvement in the presence of roselle calyx fractions. Cell-based analysis revealed the absence of host immune stimulation via Drosophila Toll pathway and roselle calyx fractions-treated immune-deficient flies lacking for components in the Toll pathway were protected from infection-induced early death phenotype and harbored reduced number of $S$. aureus colonies. Overall, our data confirmed the in vivo anti-staphylococcal activity of roselle calyx fractions in Drosophila infection model and such protective signature was devoid of host immune stimulation.
\end{abstract}

\section{Introduction}

The escalation of worldwide infection cases caused by antibiotic-resistant bacteria presents a serious challenge to the health of global population (Levy and Marshall 2004; Gelband et al. 2015). An example of such pathogenic bacteria is Staphylococcus aureus (WHO 2017), especially the strain so called Methicillin-resistant S. aureus (MRSA)(Hassoun et al. 2017). Current guideline in the treatment of $S$. aureus infection primarily relies on the application of first-line $\beta$-lactam antibiotics, such as selected penicillin and cephalosporins derivatives, and in the MRSA-related infections, vancomycin remains the first-choice arsenal to be used in the treatment (Tong et al. 2015). Unfortunately, the increasing number of $S$. aureus strains that are resistant to available antibiotics has been widely reported (Tong et al. 2015; Hassoun et al. 2017; WHO 2017), critically emphasizes the need

\footnotetext{
* Corresponding Author

E-mail Address: firzannainu@unhas.ac.id
}

to discover new antibacterial agents that are effective to treat the pathogenic mutant strains of S. aureus.

At present, directed exploration of natural resources is the main pathway to discover new entities of antibacterial agents with a possible novel mode of action (Lewis 2013; Wright 2014). Of many potential sources to find antibacterial compounds with such characteristics, roselle (Hibiscus sabdariffa L.) calyx is perhaps one of great choices. Roselle calyx extract has been reported to exert antistaphylococcal activity both in vitro (Liu et al. 2005; Alaga et al. 2014) and in vivo (Ahsan et al. 2019). With such promising results in hand, it seems sensible to advance research into the next level: step-by-step assessment on the nature of antibacterial compound(s) contained in the roselle calyx extract.

In effort to discover new antibacterial agent(s) from natural resources, the use of inexpensive and practical in vivo model organism become an important subject to consider. In accordance with that, we recently demonstrated the use of fruit fly or vinegar fly (Drosophila melanogaster) infection model to assess the 
anti-staphylococcal activity of crude extracts prepared from green algae Ulva reticulata (Nainu et al. 2018) and roselle (Hibiscus sabdariffa L.) calyx (Ahsan et al. 2019). While it is evident that the fruit fly model of infection is easy-to-use and suitable for rapid in vivo screening, this particular insect has been reported as a suitable host for Staphylococcus aureus (Needham et al. 2004; Garcia-Lara et al. 2005; Wu et al. 2012; Nainu et al. 2018). In addition, the application of costeffective yet robust techniques available in fruit fly has been central attention to the promising use of such model system in developing countries with limited access to advanced research facilities. Taking those advantages into account, here we presented the in vivo anti-staphylococcal activity of fractions prepared from ethanolic extract of roselle (H. sabdariffa L.) calyx using our established Drosophila infection model.

\section{Materials and Methods}

\subsection{Bacterial Strains and Fly Stocks}

Bacterial infection experiments were carried out using a 24-h old culture of S. aureus ATCC 29213 (collection of microbiology laboratory, Faculty of Pharmacy, Hasanuddin University). The bacterial inoculum was collected, washed carefully with phosphate buffered saline (PBS), diluted equal to 0.5 McFarland density, and suspended in PBS prior to use. The following lines of $D$. melanogaster were used as in vivo model organisms: w1118 as wildtype (background) control and $p s h[1] ; ; \operatorname{modSP}[K O]$ as the immunodeficient fly line with diminished activity of Toll pathway. Both fly lines were subjected to standard maintenance in cornmeal-agar medium at $25^{\circ} \mathrm{C}$.

\subsection{Preparation of Roselle Extract and Fractions}

Roselle (Hibiscus sabdariffa L.) calyces were collected from Makassar, South Sulawesi, Indonesia and processed in a wet and dry sortation methods. Dried samples were subsequently subjected to a maceration procedure using $70 \%$ ethanol for $1 \times 24$ hours and then re-macerated for 1 x 24 hours, as described in (Ahsan et al. 2019) with slight modifications. All resulting filtrates were pooled and processed in a rotary evaporator to produce extract with proper thickness. The resulting extract was subsequently subjected to the fractionation process using water: ethyl acetate mixture (1:1). Ethanolic extract of roselle calyces and its fractionated preparations were freezedried and used as samples in this study.

\subsection{Fly Infection, Survival Assay and Bacterial Growth Analysis}

Infection experiment was performed on the thorax of male $D$. melanogaster by using the pricking method, as described previously (Nainu et al. 2018; Ahsan et al. 2019; Nainu et al. 2019). Briefly, 4-7 days old of adult male fruit flies (30 flies per group of treatment) were pricked using a tungsten fine needle that had been previously dipped in the $S$. aureus culture (100-fold dilution of $0.5 \mathrm{McF}$ arland density). All pricked flies were maintained at $29^{\circ} \mathrm{C}$ and subjected to either assessment on fly survivorship or bacterial growth analysis using colony-forming assay as described previously (Nainu et al. 2018; Ahsan et al. 2019; Nainu et al. 2019). In the assessment of fly survival during infection, $S$. aureuspricked flies were maintained in the presence or absence of roselle fractions or $200 \mu \mathrm{g} / \mathrm{ml}$ tetracycline as positive control and the number of survived/dead flies in each group was recorded twice in a day during the course of the experiment. For the assessment of bacterial growth during infection, $S$. aureus-pricked flies were maintained in a similar fashion as in the fly survival assay and at a designated time, five live flies were collected and manually squashed in PBS solution using a micro pestle to produce a fly lysate. Homogenates prepared from each group were serially diluted and cultured on Vogel-Johnson agar medium at $37^{\circ} \mathrm{C}$ for $1 \times 24$ hours. The number of bacterial colonies found on the VogelJohnson agar plate after incubation is expressed as $\mathrm{CFU} / \mathrm{ml}$. In all fly survival and colony-forming assay experiments, groups of PBS-pricked flies were used as controls.

\subsection{In vitro Assessment on Cell Viability and Immunostimulation Potency}

Cell viability and immunostimulation potency were assessed using cell-based reporter assay system, as described previously (Kanoh et al. 2015). Briefly, Drs-LucDL1 Drosophila cell line was incubated in the presence of tested samples at six different concentrations starting from $0.1 \mathrm{ppm}$ to $100 \mathrm{ppm}$. Drs-Luc-DL1 Drosophila cells were tested for the viability upon incubation with different samples' concentrations for 12 hours at $25^{\circ} \mathrm{C}$ using CellTiter ${ }^{\circledR}-G l o$ Luminescent Cell Viability Assay (Promega) according to the manufacturer's protocol. Using the same experimental layout, Drs-Luc-DL1 Drosophila cells were examined for the Drosomycin stimulation potency using ONE-Glo Luciferase Assay System (Promega) according to the manufacturer's protocol.

\subsection{Antimicrobial Peptide (AMP) Expression Analysis}

Analysis on the expression of Drosomycin, an endogenous antimicrobial peptide produced by Drosophila in response to Gram-positive bacterial infection, was carried out using total RNA prepared from five $w^{1118}$ fly line in each experimental group at 
50 hours post-inoculation with S. aureus. The collected Drosophila were homogenized in the Treff tube and subsequently processed using SV Total RNA Isolation System (Promega) as per manufacturer's instruction. Assessment on the level of Drosomycin (Drs) was carried out based on the reverse transcriptase (RT)qPCR method using a pair of $D r s$ primer. The sequence of Drs forward (F) and Drs reverse (R) were as follows: (Drs-F) 5'-CGTGAGAACCTTTTCCAATATGATG-3' and (Drs-R) 5'-TCCCAGGACCACCAGCAT-3'. All RT-qPCR reactions were held in a $20 \mu$ volume using GoTaq ${ }^{\circledR}$ 1-Step RT-qPCR System (Promega), according to the manufacturer's protocol. The following RT-qPCR profile was carried out in a Rotor-Gene $Q$ thermal cycler (Qiagen, Germany): reverse transcription step at $37^{\circ} \mathrm{C}$ for 15 mins, denaturation step at $95^{\circ} \mathrm{C}$ for 10 mins, and 40 cyclic repeats of $95^{\circ} \mathrm{C}$ for 10 secs and $60^{\circ} \mathrm{C}$ for 30 secs, and final extension step at $72^{\circ} \mathrm{C}$ for 30 secs. The expression level of host reference gene, ribosomal protein $r p 49$, was assessed in each run using a pair of rp49 primer and using the same RT-qPCR protocol as above. The sequence of $r p 49$ forward $(F)$ primer and $r p 49$ reverse (R) primer were as follows: (rp49-F) 5'-GACGCTTCAAGGGACAGTATCTG-3' and (rp49-R) 5'-AAACGCGGTTCTGCATGAG-3'. The relative expression of Drs to rp49 was analyzed using qGENE software (version 2.0.3.2) and the result was further subjected to statistical analysis. To verify the specificity of primers used in our RT-qPCR experiments, standard melt curve analysis was performed in each run.

\subsection{Data Processing, Graph Preparation, and Statistical Analysis}

All data, obtained from at least three independent experiments, were statistically processed using GraphPad Prism ${ }^{\circledR}$ 8. Data on fly survivorship were constructed as a Kaplan-Meier graph and statistically analyzed using log-rank approach. Data on bacterial growth, cell viability, immune stimulation potency, and Drosomycin mRNA level were shown as bar graphs and subjected to statistical analysis using one-way ANOVA. All data presented in this study are shown as mean \pm S.D and $\mathrm{p}$ values of less than 0.05 were considered as significant.

\section{Results}

\subsection{Dose-dependent Toxicity of Roselle Fractions in Cell Culture and Adult of Drosophila melanogaster}

In this study, we investigated the antistaphylococcal effect of water and ethyl acetate fractions, or simply abbreviated as WFR and EAFR respectively, prepared from ethanolic extract of roselle calyx using our established Drosophila-based in vivo approach. As a start, we prepared WFR and EAFR from the ethanolic extract of roselle calyces and subsequently performed cell viability assay and fly survival assay to determine the toxicity as well as an appropriate concentration of WFR and EAFR to be used in the further experiments. As shown in Figure 1a, the viability of Drosophila cells was negatively affected by both samples at high concentrations (250 ppm) but not at the lower concentrations tested in the experiments. In accordance to that, the survival of adult male flies of $w^{1118}$ (Figure 1b) and psh[1];;modSP[KO] immunodeficient mutant line (Figure 1c) were not affected upon ingestion of either WFR or EAFR at concentrations up to $2 \% \mathrm{w} / \mathrm{w}$. However, the survivals of both fly lines were adversely affected once maintained in foods containing each fractionated roselle samples at higher concentration $(8 \% \mathrm{w} / \mathrm{w})$. Results obtained from both in vitro and in vivo experiments clearly suggested the dose-dependent lethal effect of roselle fractions used in this study. To rule out such possible toxic influence, we used WFR and EAFR at a concentration ranging from 100 to 0.1 $\mathrm{ppm}$ and at concentration of $0.5 \%$ and $2 \%(\mathrm{w} / \mathrm{w})$ in further in vitro and in vivo experiments, respectively.

\subsection{Augmentation of Host Survival Rate by Roselle Fractions During Bacterial Infection}

We previously reported the antibacterial activity of roselle calyx crude extract against Gram-positive $S$. aureus in D. melanogaster. However, the nature of the antibacterial compounds remains unidentified. To provide better insight, we carried out similar in vivo fly survival and CFU assays, in addition to an in vitro approach firstly performed in this study, using WFR and EAFR, fractions prepared from roselle calyx extract. We found that the survival of adult $D$. melanogaster $w^{1118}$ was rapidly declined under $S$. aureus infection condition and such trend was subject for improvement by the use of either antibiotic (as positive control) or the crude extract of roselle calyx (5\%). Treatments of S. aureus-infected $w^{1118}$ with WFR or EAFR at a concentration of $2 \%$, but not at $0.5 \%$, were sufficient to reduce flies' mortality (Figure 2a), suggesting the in vivo anti-staphylococcal action of roselle fractions, WFR and EAFR, at a suitable concentration.

\subsection{Inhibition of Bacterial Growth by Roselle Fractions}

Augmentation of flies' mortality during S. aureus infection was reported to be linked with amplified bacterial load. Hence, the inhibition of bacterial proliferation, either by the use of antibiotics or crude plant extracts, has been shown to enhance the survivorship of infected Drosophila. With this in mind, 

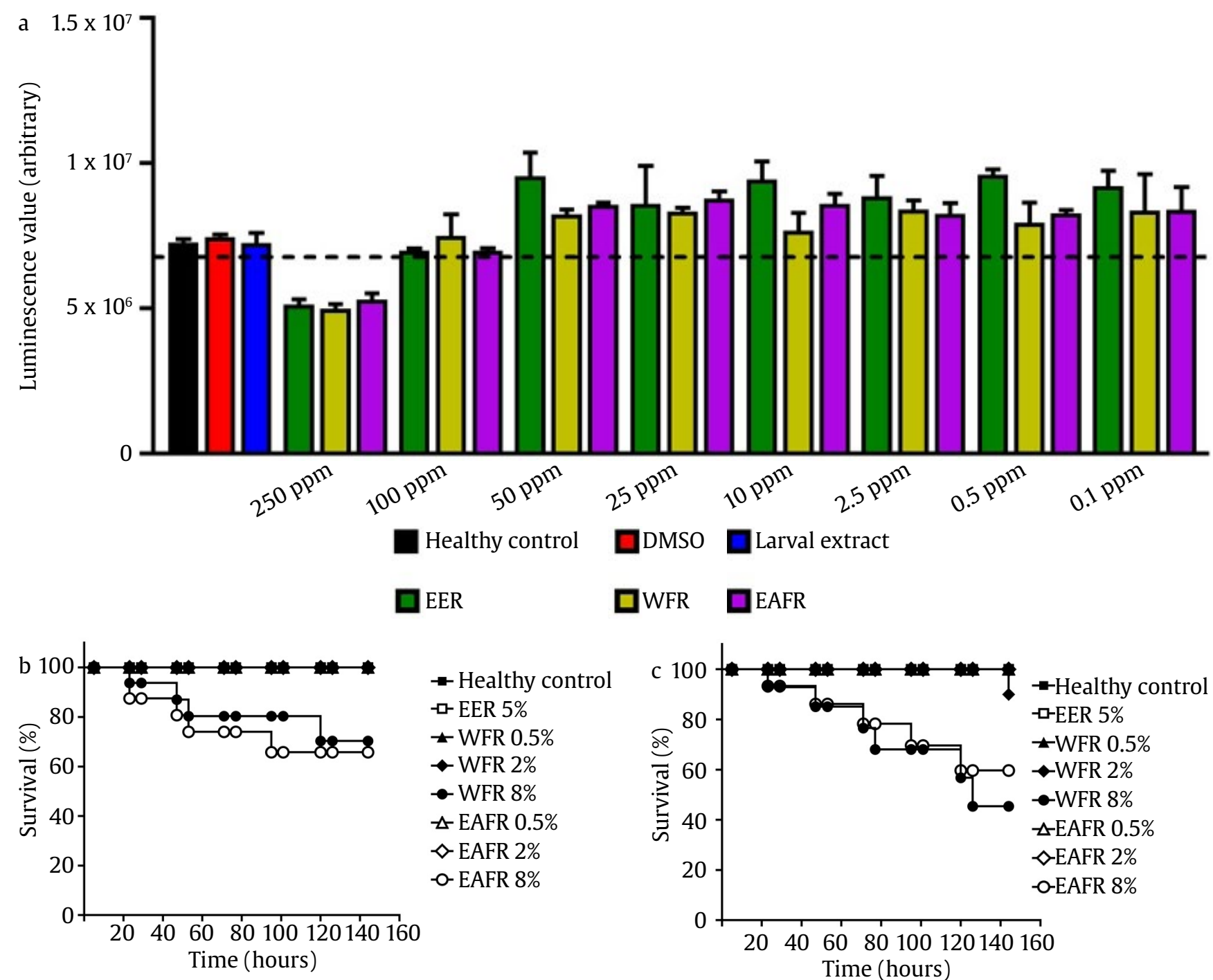

Figure 1. Dose-dependent toxicity of roselle fractions in Drosophila. (a) The incorporation of either WFR or EAFR in the cell media reduced the viability of Drs-Luc-DL1 cells in a concentration-dependent manner. (b and c) Similarly, ingestion of food-containing either WFR or EAFR decreased the survivorship of flies in a manner dependent on samples' concentration. Adult 4-7 days old male $w^{1118}$ (b) and psh[1];; $\operatorname{modSP}[K O]$ (c) were maintained at $25^{\circ} \mathrm{C}$ in fly food containing samples at three different concentrations $(0.5,2$, and $8 \%)$. The number of survived and dead flies were recorded daily and fly survivorship was analysed using a Kaplan-Meier-Log Rank approach. EER: ethanolic extract of roselle calyx, WFR: water fractions of rosella calyx extract, EAFR: ethyl acetate fractions of rosella calyx extract

we carried out CFU assays to confirm whether protection seen in both WFR-treated and EAFR-treated S. aureusinfected flies (Figure 2a) was the result of bacterial growth inhibition. Indeed, we noticed that continuous treatment of infected Drosophila with either 2\% WFR or $2 \%$ EAFR was surely effective to reduce bacterial growth (Figure $2 \mathrm{~b}$ ), further maintaining the notion that inhibition of bacterial growth is vital in order to achieve improved host survivorship during infection.

\subsection{Anti-Staphylococcal Activity of Roselle Fractions was Achieved Independent of Drosomycin Expression}

Drosophila expressed an array of antimicrobial peptides (AMPs), including the well-characterized Drosomycin, that plays an important role in the innate immune response against $S$. aureus. To determine the involvement of Drosomycin expression in the antibacterial activity of WFR and EAFR against S. aureus, 

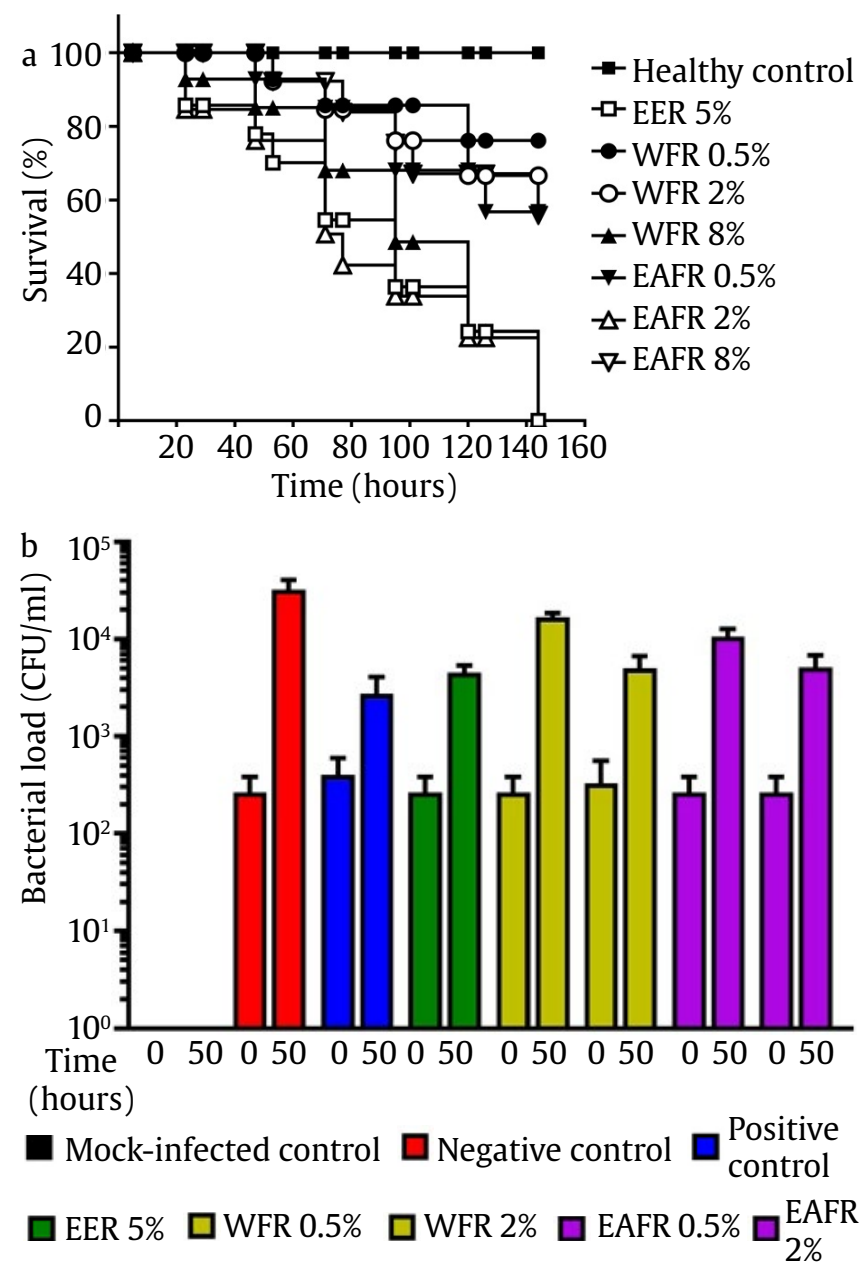

Figure 2. Increased fly survivorship and impaired bacterial proliferation in infected flies given either WFR or EAFR. Adult 4-7 days old male $w^{1118}$ were pricked with $S$. aureus and maintained at $25^{\circ} \mathrm{C}$ in food containing samples at two different concentrations ( 0.5 and $2 \%)$. These flies were subjected to survival assay (a) and analysis of bacterial growth (b). Groups of flies infected with $S$. aureus and maintained in the presence or absence of $200 \mu \mathrm{g} / \mathrm{ml}$ tetracycline were used as the negative and positive control groups, respectively. EER: ethanolic extract of roselle calyx, WFR: water fractions of rosella calyx extract, EAFR: ethyl acetate fractions of rosella calyx extract

we carried out two experimental approaches: in vitro and in vivo. In the in vitro experiment, we assessed Toll pathway-mediated Drosomycin expression by using cell-based reporter assay. We found that the expression of Drosomycin was not increased upon treatment with either WFR or EAFR (Figure 3a). In conjunction with the in vitro result, we also assessed the expression level of Drosomycin by RT-qPCR method using lysates prepared from adult $w^{1118}$ flies. As shown in Figure 3b, Drosomycin (Drs) expression was considerably induced in response to $S$. aureus infection and a comparable level of gene expression was similarly observed in other fly groups: the control groups (tetracycline-treated or $5 \%$ roselle calyx extract-treated), WFR-treated, and EAFRtreated S. aureus-infected fly groups. Moreover, level of Drs expression in all groups of treatments was not significantly different, clearly indicating that treatments given to the infected flies, either antibiotics, $5 \%$ roselle extract, $2 \%$ WFR, or 2\% EAFR, did not increase the expression of Drs thus had no effect on the stimulation of Drs-related innate immune response in the S. aureusinfected host.

\subsection{Protective Effect of Roselle Fractions in the S. aureus-infected Immunodeficient Host}

Increased fly survivorship and reduced bacterial load were two trends observed in groups of S. aureusinfected flies that were incubated in the presence of roselle fractions, WFR or EAFR. These two protection signatures were achieved by mechanisms independent of Drosomycin, one of the most important AMPs expressed by Drosophila in response to $S$. aureus infection. Therefore, we anticipated that the antistaphylococcal effect of WFR and EAFR might be resulted from the presence of selected antibacterial compounds available at a certain concentration in the roselle fractions tested in this study. To confirm this, we carried out pricking-based infection experiments on the psh[1];; $\operatorname{modSP}[K O]$, a fly line lacking for Psh and ModSP, two important components in the canonical Toll-mediated antibacterial immune response against S. aureus. As illustrated in Figure 4, the survival of psh[1];;modSP[KO] mutant flies was declined in a faster rate (Figure 4a) and infected immunodeficient mutant flies harbored slightly higher bacterial load (Figure $4 \mathrm{~b}$ ) than its wild-type counterpart (Figure $2 \mathrm{a}$ and b, respectively), indicating the vital role of Psh and/or ModSP in the canonical Toll-mediated immune response against $S$. aureus infection. Nonetheless, it is important to note that two protection signatures: improvement of mutant flies' survivorship (Figure 4a) and reduction of bacterial growth (Figure $4 \mathrm{~b}$ ) were evident in the groups of infected mutant flies upon treatment with either $2 \%$ WFR or $2 \%$ EAFR. Taken together, our results confirmed the in vivo anti-staphylococcal activity of $2 \%$ WFR and 2\%EAFR in the wildtype and immunodeficient Drosophila infection model. 

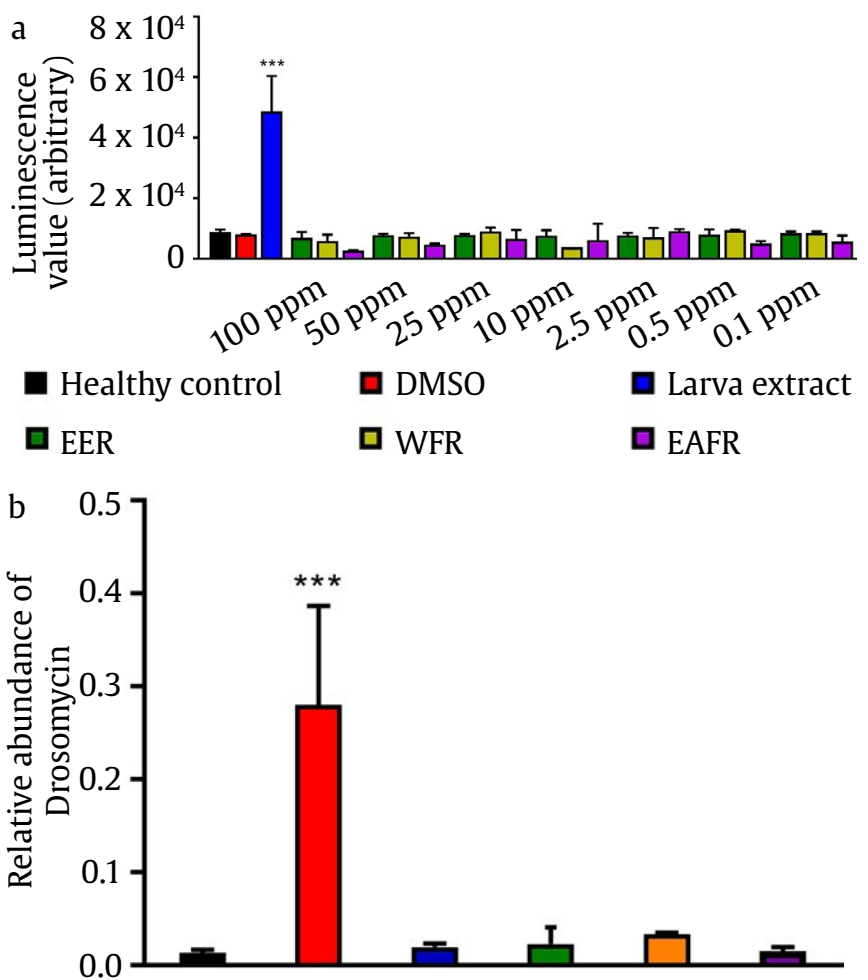

Mock-infected control $\square$ Negative control

Positive control $\square$ EER 5\% $\square$ WFR 2\% $\square$ EAFR 2\%

Figure 3. Anti-staphylococcal protection of either WFR or EAFR was not due to stimulation in host humoral immunity. (a) Drs-Luc-DL1 cell line was treated with samples at different concentrations and subsequently assessed for its Drs level based on luciferase activity $(\mathrm{p}<0.001)$. (b) Adult 4-7 days old male $w^{1118}$ were pricked with $S$. aureus and maintained at $25^{\circ} \mathrm{C}$ in food containing samples at given concentrations. These flies were subjected to total RNA isolation and subsequently followed by RT-qPCR for quantification of Drosomycin mRNA level. The expression level of $r p 49$ was used as the reference control $(\mathrm{p}<0.001)$. EER: ethanolic extract of roselle calyx, WFR: water fractions of rosella calyx extract, EAFR: ethyl acetate fractions of rosella calyx extract

\section{Discussion}

Fruit fly $D$. melanogaster has been widely used to investigate cellular and molecular events that are relatively difficult to be addressed in higher model organisms (Pandey and Nichols 2011; Ugur et al. 2016; Nainu et al. 2017; Rahmatika et al. 2019). In fact, in the last decade, low- to high-throughput drug discovery using $D$. melanogaster platform has been widely performed (Willoughby et al. 2013; Fernández-Hernández et al. 2016; Ekowati et al. 2017),
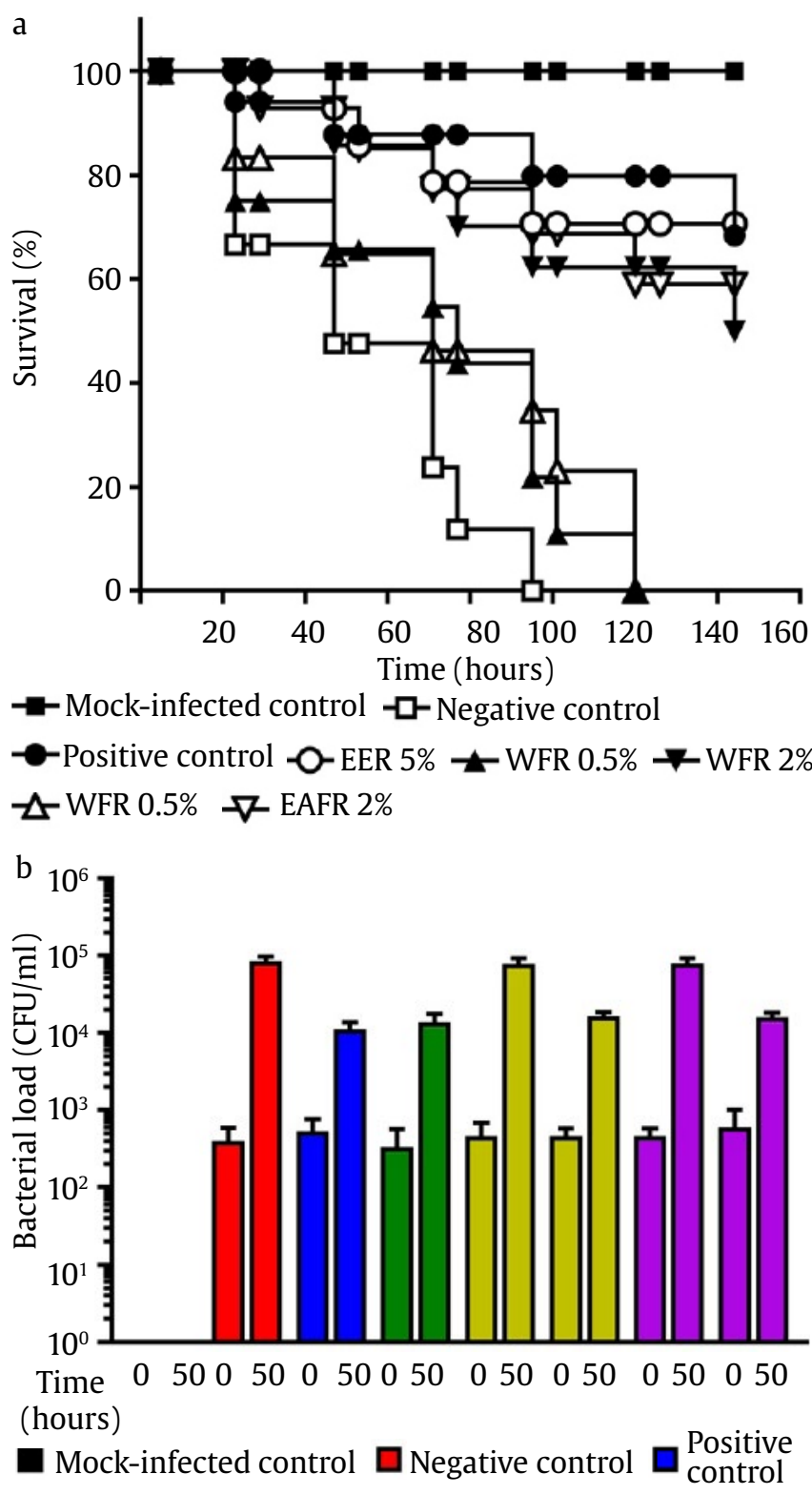

EER 5\% $\square$ WFR 0.5\% $\square$ WFR 2\% $\square$ EAFR 0.5\% $\square_{2 \%}^{\text {EAFR }}$

Figure 4. Improved host survivorship and decreased bacterial growth in infected immunodeficient $\operatorname{psh}[1] ; ; \operatorname{modSP}[K O]$ flies in the presence of WFR or EAFR. Adult 4-7 days old male $p s h[1] ; ; \bmod S P[K O]$ flies were pricked with $S$. aureus and maintained at $25^{\circ} \mathrm{C}$ in food containing either water or ethyl acetate fractions of roselle extract. These flies were subsequently subjected to survival assay (a) and analysis of bacterial growth (b). Groups of flies infected with S. aureus and maintained in the presence or absence of $200 \mu \mathrm{g} / \mathrm{ml}$ tetracycline were used as the negative and positive control groups, respectively. EER: ethanolic extract of roselle calyx, WFR: water fractions of rosella calyx extract, EAFR: ethyl acetate fractions of rosella calyx extract 
and interestingly, one drug (Vandetanib, ZD6474) was successfully approved by US FDA for the treatment of medullary thyroid carcinoma in 2011 (Das and Cagan 2013).

In this study, we examined the antibacterial effect of two roselle fractions, WFR and EAFR, against $S$. aureus using a simple yet robust in vitro and in vivo pharmacological approach based on the use of D. melanogaster. A major advantage of our in vivo approach is the applicability of several important assays that are time-consuming or difficult to be performed using vertebrate model organisms. In terms of infection experiment, some of the experimental features such as simplicity of host survival assessment during the course of infection, easiness to perform colony-forming assay or gene expression analysis using whole-body samples or in the particular sites of infected tissues, and more importantly, broad availability of various readyto-use lines of immunodeficient fruit flies, remain impressive powerful traits to be signified in Drosophila model of infection (Chamilos et al. 2011; Tzelepis et al. 2013).

The antibacterial effect of roselle fractions, WFR and EAFR, against $S$. aureus infection in Drosophila is likely to be achieved independent from additional participation of canonical Toll-mediated innate immune response in the infected host, as suggested by results obtained from $p s h[1] ; ; \operatorname{modSP}[\mathrm{KO}]$ fly line that was used as immunodeficient Drosophila model. In the absence of two important components of Toll pathway, Psh and ModSP, stimulation of humoral innate immunity in response to $S$. aureus infection in Drosophila (i.e. the expression of AMPs) are impaired thus leads to an immunodeficient-like state (Buchon et al. 2014). Flies with such state has been shown to succumb faster during pathogen challenge than their wild-type counterparts (El Chamy et al. 2008; Buchon et al. 2009). However, in our study, we demonstrated that ingestion of either WFR or EAFR could improve the survivorship of infected psh[1];; $\operatorname{modSP}[$ KO] fly line, suggesting the anti-staphylococcal nature of roselle fractions.

Prior to infection experiments, we carried out toxicity assays using either cell cultures (in vitro) or adult flies (in vivo). Data obtained in these straightforward and time-effective assays suggested the toxicity of both roselle fractions was yielded in a manner dependent on the given dose of samples. Such results were important to decide the proper dose of roselle fractions to be used in the next experiments and to rule out the possible toxic effect of samples to host survival during infection experiments.

In this research, we extended our previous results to demonstrate the in vivo anti-staphylococcal effect of fractions prepared from ethanolic extract of roselle calyx using a Drosophila platform system. While our in vitro and in vivo results were in line, it is important to note that in vivo testing of drug candidate(s) could provide better insight than the in vitro results in terms of potential efficacy as well as possible harmful effect of drug candidate(s) to individuals, especially in the case where the tested compound(s) are pro-drug. Hence, the use of inexpensive in vivo model organism, such as D. melanogaster (Pandey and Nichols 2011), that bids a high chance for getting similar outcomes in the clinical trial on human subjects could be a feasible option to improve our traditional drug discovery pipeline.

\section{Conflict of Interest}

We declare that we have no conflict of interest.

\section{Acknowledgements}

We would like to thank to all members of Unhas Fly Research Group for their kind help in the fly maintenance, reagents preparation, and technical assistance. We also would like to offer our gratitude to Dr. Bruno Lemaitre for the immunodeficient fly line. This research was financially supported by Penelitian Dasar UNHAS (PDU) 2019 research grant (No. 641/ UN4.1/KEP/2019) from Hasanuddin University (to MND, FN, and Su) and by JSPS KAKENHI Grant and The Naito Foundation (to TK). The use of FlyBase is acknowledged.

\section{References}

Ahsan $\mathrm{M}$ et al. 2019. In vivo anti-staphylococcal activity of roselle (Hibiscus sabdariffa L.) calyx extract in Drosophila model of infection, J HerbMed Pharmacol 8:41-46.

Alaga TO et al. 2014. Phytochemical and in vitro anti-bacterial properties of Hibiscus sabdariffa L (Roselle) juice. $J$ Med Plant Res 8:339-344.

Buchon $\mathrm{N}$ et al.. 2009. A single modular serine protease integrates signals from pattern-recognition receptors upstream of the Drosophila Toll pathway. Proc Natl Acad Sci USA 106:12442-12447.

Buchon N et al.. 2014. Immunity in Drosophila melanogasterfrom microbial recognition to whole-organism physiology. Nat Rev Immunol 14:796-810. 
Chamilos G et al. 2011. Drosophila melanogaster as a model host for the study of microbial pathogenicity and the discovery of novel antimicrobial compounds. Curr Pharm Des 17:1246-1253.

Das TK, Cagan R. 2013. A Drosophila approach to thyroid cancer therapeutics. Drug Discov Today Technol 10:6571.

El Chamy L et al.. 2008. Sensing of 'danger signals' and pathogen-associated molecular patterns defines binary signaling pathways 'upstream' of Toll. Nat Immunol 9:1165-1170.

Ekowati H et al. 2017. Protective effects of Phaseolus vulgaris lectin against viral infection in Drosophila. Drug Discov Ther 11:329-335.

Fernández-Hernández I et al. 2016. The translational relevance of Drosophila in drug discovery. EMBO rep 17:471-472.

García-Lara J et al. 2005. Invertebrates as animal models for Staphylococcus aureus pathogenesis: a window into host-pathogen interaction. FEMS Immunol Med Microbiol 43:311-323.

Gelband H et al. 2015. The state of the world's antibiotics 2015. The Center for Disease Dynamics, Economics and Policy. Washington: CDDEP.

Hassoun A et al. 2017. Incidence, prevalence, and management of MRSA bacteremia across patient populations-a review of recent developments in MRSA management and treatment. Crit Care 21:211.

Kanoh $\mathrm{H}$ et al. 2015. Ex vivo genome-wide RNAi screening of the Drosophila Toll signaling pathway elicited by a larva-derived tissue extract. Biochem Biophys Res Commun 467:400-406.

Levy SB, Marshall B. 2004. Antibacterial resistance worldwide: causes, challenges and responses. Nat Med 10:122-129.

Lewis K. 2013. Platforms for antibiotic discovery. Nat Rev Drug Discov 12:371-387.

Liu KS et al. 2005. In vitro antibacterial activity of roselle calyx and protocatechuic acid. Phytother Res 19:942-945.
Nainu F etal. 2017. Induction of apoptosis and subsequent phagocytosis of virus-infected cells as an antiviral mechanism. Front Immunol 8:1-11.

Nainu $\mathrm{F}$ et al. 2018. In vivo antibacterial activity of green algae Ulva reticulata against Staphylococcus aureus in Drosophila model of infection. Pharmacog J 10:993997.

Nainu F et al. 2019. Protective effect of green algae Ulva reticulata against Pseudomonas aeruginosa in Drosophila infection model. HAYATI J Biosci 26:163171.

Needham AJ et al. 2004. Drosophila melanogaster as a model host for Staphylococcus aureus infection. Microbiol 150:2347-2355.

Pandey UB, Nichols CD. 2011. Human disease models in Drosophila melanogaster and the role of the fly in therapeutic drug discovery. Pharmacol Rev 63:411436.

Rahmatika D et al. 2019. Inhibitory effects of viral infection on cancer development. Virology 528:48-53.

Tong SYC et al. 2015. Staphylococcus aureus infections: Epidemiology, pathophysiology, clinical manifestations, and management. Clin Microbiol Rev. 28:603-661.

Tzelepis I et al. 2013 Drosophila melanogaster: a first step and a stepping-stone to anti-infectives. Curr Opin Pharmacol. 13:763-768.

Ugur B et al. 2016. Drosophila tools and assays for the study of human diseases. Dis Mod Mech 9:235-244.

Willoughby LF et al. 2013. An in vivo large-scale chemical screening platform using Drosophila for anti-cancer drug discovery. Dis Mod Mech 6:521-529.

Wright GD. 2014. Something old, something new: revisiting natural products in antibiotic drug discovery. Can J Microbiol 60:147-154.

$\mathrm{Wu} \mathrm{K}$ et al. 2012. Assessment of virulence diversity of methicillin-resistant Staphylococcus aureus strains with a Drosophila melanogaster infection model. BMC Microbiol 12:274. 